\title{
BIOFILM FORMATION AND QUORUM SENSING ANALYSIS OF UROPATHOGENIC MULTIDRUG RESISTANCE ESCHERICHIA COLI
}

\author{
MOHANA PRIYA M. ${ }^{1}$, G. BHUVANESHWARI ${ }^{2}$ \\ 1,2Department of Microbiology, Saveetha Medical College and Hospital, Chennai 602105 \\ Email: manipriyamedmic@gmail.com
}

Received: 12 Nov 2020, Revised and Accepted: 10 Jan 2021

\begin{abstract}
Objective: Escherichia coli (E. coli) are gram-negative facultative anaerobes which are commonly found in the lower intestine. Biofilm production in E. coli promotes colonization and leads to an increase rate of infections, and such infections may be difficult to treat as they exhibit multidrug
\end{abstract} resistance (MDR).

Methods: 50 strains of uropathogenic E. coli were collected from Clinical Microbiology laboratory at Saveetha medical college and hospital for a time period of 3 mo. Strains were identified by conventional biochemical methods. Biofilm formation and quorum sensing analysis were performed by the Microtitre plate method and Thin Layer Chromatography method (TLC), respectively.

Results: In this study, 46 (92\%) of E. coli strains were strong, 3(6\%) were intermediate and 1(2\%) were weak biofilm producers. From TLC analysis, $34(68 \%)$ of the strains produced Acyl Homoserine Lactone molecules. Out of which, 16 isolates were shown unknown analytes of Retardation factor (Rf) value greater than 1. The Rf values identified were 3 unsubstituted C4 (5), 3 unsubstituted C6 (3), 3 oxo C8 (3), 3 oxo C4 (4), 3 oxo C6 (2), 3 oxo C1 (1).

Conclusion: In this study, 100\% of isolates were biofilm producers. Of which 18 strains produced known Acyl Homoserine Lactone molecules and 16 isolates produced unknown analytes. Thus, quorum sensing molecules plays a major role in biofilm formation.

Keywords: Biofilm formation, Quorum sensing, Uropathogenic E. coli, Acyl Homoserine Lactone

(C) 2021 The Authors. Published by Innovare Academic Sciences Pvt Ltd. This is an open access article under the CC BY license (https://creativecommons.org/licenses/by/4.0/) DOI: https://dx.doi.org/10.22159/ijcpr.2021v13i2.41549. Journal homepage: https://innovareacademics.in/journals/index.php/ijcpr

\section{INTRODUCTION}

E. coli is a type of bacteria that normally lives in the intestine. UTI is a serious health problem with respect to antibiotic resistance and biofilm formation being the prime cause for antibiotic resistance. Antibiotics are commonly used to prevent bacterial infection and diseases for many decades since their discovery at the beginning of the 20th century. However, emerging evidence [1] indicates that traditional antibiotic treatments tend to be ineffective for the patients due to the emergence of drug-resistant pathogens resulting from antibiotics overuse [2, 3].

According to national institutes of health, biofilms are responsible for $>60 \%$ of all microbial infections $[4,5]$; the biofilms produced by $E$. coli may be difficult to treat as they exhibit multidrug resistance. Biofilm prevalence among uropathogenic. E. coli ranges from $60 \%$ $70 \%$. Biofilm formation also increases the risk of recurrent UTI [6]. This can be reduced by appropriate treatment with antibiotics like Fluroquinolone, cephalosporin and aminoglycoside. Quorum sensing is a bacterial cell signaling which allows both gram-negative and gram-positive bacteria to sense one another.

Quorum sensing is done with the help of autoinducers such as acyl homoserine lactone (ahl-1). Acyl synthase (LUX I) is involved in the release of this autoinducer [7].

Biofilm production in Escherichia coli promotes colonization and leads to an increased rate of infections. Such infections may be difficult to treat as they exhibit multidrug resistance (MDR).

The present study is aimed to perform in-vitro detection of biofilm formation and quorum sensing among uropathogenic $E$. coli strains from urine cultures by Microtitre plate method and Thin Layer Chromatography method (TLC), respectively.

\section{MATERIALS AND METHODS}

The present study was conducted in the Department of Microbiology at Saveetha medical college, Chennai, India. The ethical clearance has been obtained for the study using conventional methods, a total number of 50 uropathogenic E. coli from the clinical samples were isolated.

Primary inoculation of the samples was done on MacConkey Agar Plate (M. A), Nutrient Agar Plate (N. A) and Blood Agar Plate (B. A). After inoculation, these samples were incubated aerobically at $37^{\circ} \mathrm{C}$ for $24 \mathrm{~h}$. Plates showing no growth during the first $24 \mathrm{~h}$ were further incubated for the next $24 \mathrm{~h}$. Antibiotic susceptibility test was done by Kirby Bauer disc diffusion method as per CLSI (clinical and laboratory standards institute) guidelines [8]. Multidrug resistance E. coli strains were collected and done biofilm formation by microtitre plate method [9]. Quorum sensing analysis $(5,6)$ was performed by the thin-layer chromatography method (tlc).

\section{Biofilm formation}

\section{Microtitre plate method}

Isolates were inoculated in trypticase soy broth and incubated for $24 \mathrm{~h}$ at $37{ }^{\circ} \mathrm{C}$ then diluted with fresh trypticase soy broth in 1:100 dilution. Tissue culture plate, which is sterile polystyrene 96 well flat bottom is filled with $0.2 \mathrm{ml}$ aliquots of the diluted culture. The broth served as control to check sterility and non-specific binding of media. The microtitre plate was incubated for $24 \mathrm{~h}$ at $37{ }^{\circ} \mathrm{C}$. By tapping the plates, the content of each well was gently removed and washed 4 times with $0.2 \mathrm{ml}$ Phosphate buffered saline to remove free-floating planktonic bacteria, Wells were stained with crystal violet. To remove excess stain wells were washed with deionized water and the tubes were dried. [10] Optical density (OD) was determined at a wavelength of $570 \mathrm{~nm}$ with micro ELISA auto reader. Experiment was repeated thrice and standard deviation was calculated. The mean OD value obtained from media control was deducted from all the test OD value.

\section{RESULTS}

In this study 50 uropathogenic multidrug resistance, E. coli strains were collected and processed using the conventional method. Culture characteristics were analyzed. 


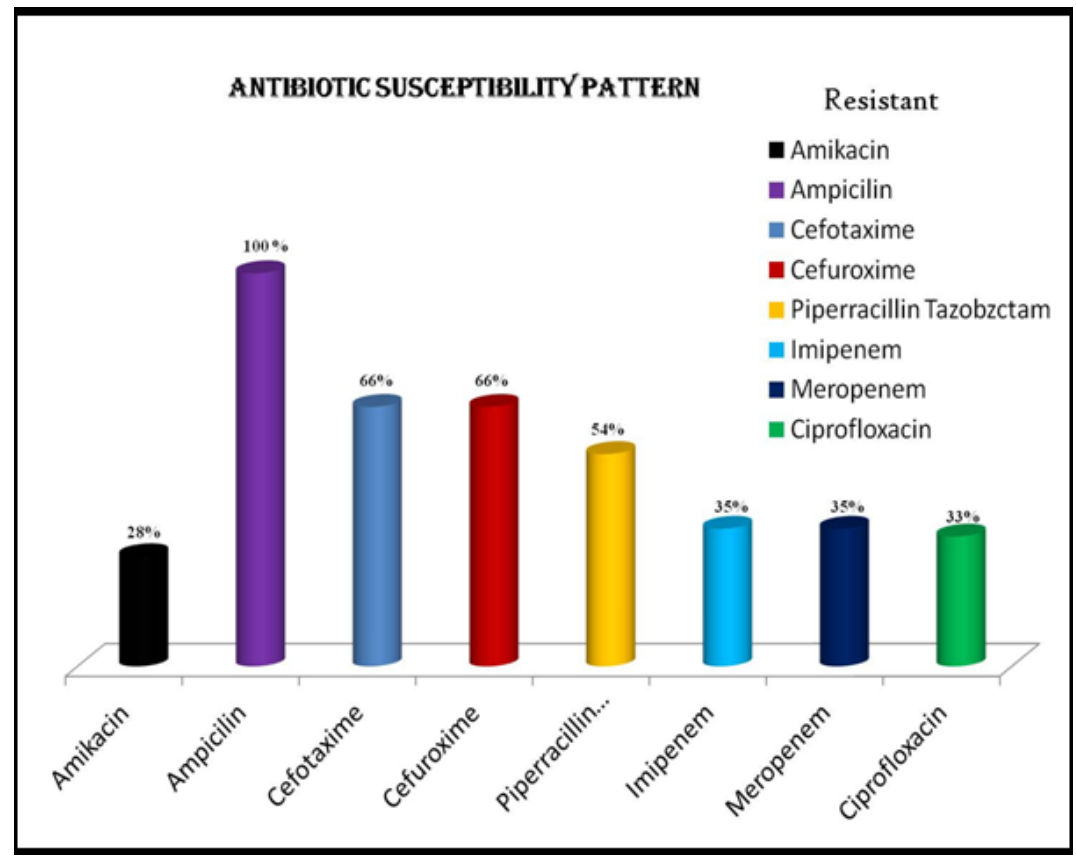

Fig. 1: Graphical representation of antibiotic susceptibility pattern

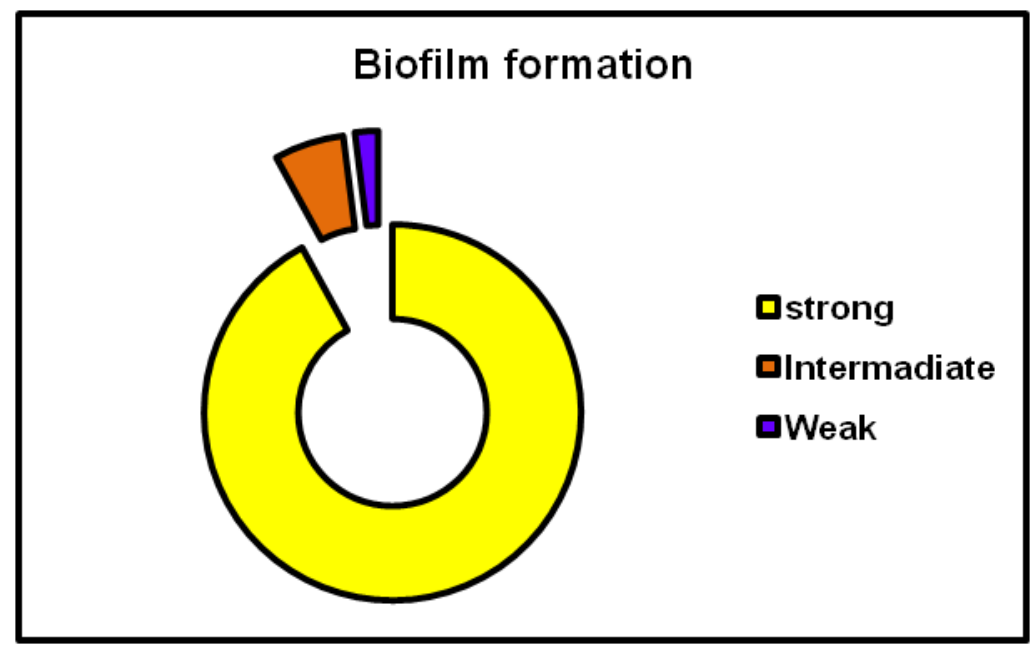

Fig. 2: Biofilm formation

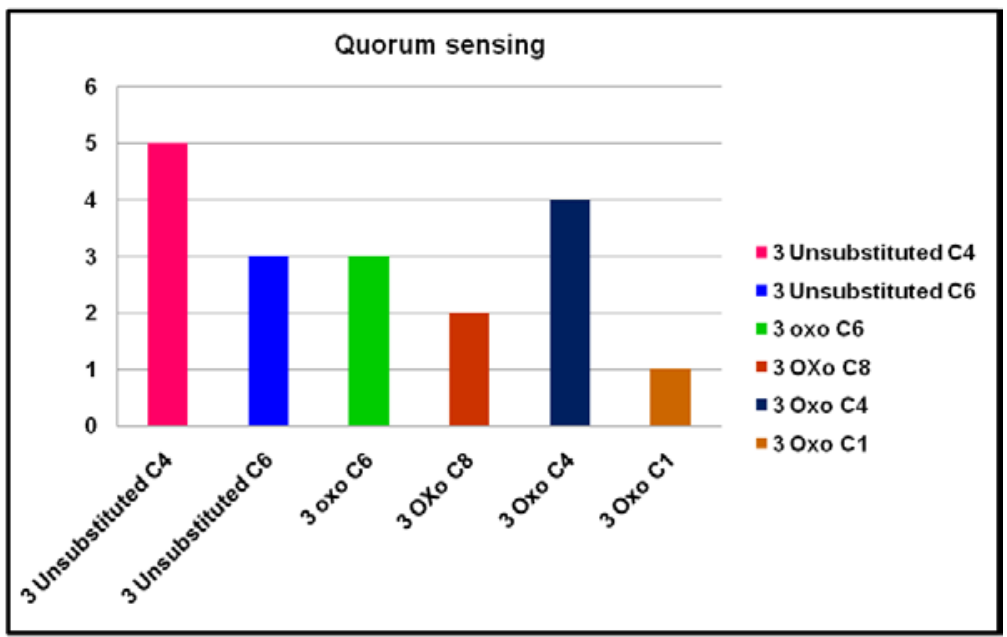

Fig. 3: Graphical representation of quorum sensing 


\section{Antibiotic susceptibility test}

On analyzing ABST the following resistant patterns were observed:

Amikacin-28\%, Ampicillin-100\%, Ciprofloxacin-33\%, Cefotaxime 66\%,Cefuroxime-66\%, Piperacillin/Tazobactam-54\%, Imipenem$35 \%$, Meropenem-35\%. Fig. 1 shows the graphical representation of Antibiotic susceptibility Pattern.

\section{Biofilm formation}

Out of 50 uropathogenic multidrug resistance $E$. coli strains, 46 (92\%) strains were strong, $3(6 \%)$ were intermediate and $1(2 \%)$ was weak biofilm producers. Fig. 2: Shows the biofilm formation.

\section{Thin-layer chromatography method (TLC)}

From TLC analysis, 34 (68\%) of the strains produced acylhomoserine lactone molecules. Out of which, 16 isolates were shown unknown analytes of retardation factor ( $\mathrm{rf}$ ) value greater than 1 . The rf values identified were 3 unsubstituted C4 (5), 3 unsubstituted C6 (3), 3 oxо C8 (3), 3 oxо C4 (4), 3 oxо C6 (2), 3 oxo C1 (1). Fig.3: shows the graphical representation of quorum sensing fig. 3: Shows the graphical representation of quorum sensing

\section{DISCUSSION}

The present study was conducted in the Department of Microbiology at Saveetha medical college, Chennai, India. The ethical clearance has been obtained for the study using conventional methods, a total number of 50 uropathogenic $E$. coli from the clinical samples were isolated.

In the present study, antibiotic resistance of biofilm producing E. coli was found to be $100 \%$ for Ampicillin, $66 \%$ for Cefuroxime and Cefotaxime, 54\% for Piperacillin/Tazobactam, 35\% for Imipenemand and Meropenem, 28\% for Amikacin, 33\% for Ciprofloxacin. In another study, antibiotic resistance of biofiom producing E. coli were found to be $100 \%$ for chloramphenicol and Amoxyclav (amoxicillin-clavulanic acid), $86 \%$ for gentamicin and cefotaxime, $84 \%$ for ceftazidime, $83 \%$ for cotrimoxazole and Piperacillin/Tazobactam, 75\% for tetracucline, 70\% for amikacin showed by poovendran ponnusamy et al., [11]

In the persent study, all the $100 \%$ E. coli strains were biofilm producers.46(92\%) strains were strong, 3(6\%) were intermediate and $1(2 \%)$ was weak biofilm producers.

Elahe Tajbakhsh et al., showed that $61.53 \%$ were able to make biofilm that $15(18.75 \%)$ isolates indicated strong reaction, $20(25 \%)$ of medium and $45(56.25 \%)$ of weak biofilm reaction [12].

Another study which was done by Mittal et al., showed that among $100(60.2 \%)$ strains, 72 strains displayed a biofilm positive phenotype and showed $6.6 \%$ strains were highly positive, $80.8 \%$ were moderate positive and $14.14 \%$ were weakly positive [13]. A study conducted by Rohollah Taghadosi et al., [14] shows the biofilm formation assay identified $10(28.57 \%)$ isolates with strong, $16(45.71 \%)$ with moderate, and 9 (25.71\%) with weak biofilm activities.

\section{CONCLUSION}

Biofilm production makes the organism to be more resistant to antibiotics and virulent as compared to non-biofilm producers. $68 \%$ Uropathogenic Escherichia coli produces quorum sensing analytes which in turn can induce other strains to be Multidrug-resistant in the future. This needs effective surveillance and control measures for preventing Healthcare-associated infections in the future.

\section{FUNDING}

Nil

\section{AUTHORS CONTRIBUTIONS}

All the authors have contributed equally.

\section{CONFLICTS OF INTERESTS}

Declared none

\section{REFERENCES}

1. LA Barton, MW Simon. Prophylactic antibiotics: Ineffective or inefficacious. Clin Pediatr 2014;53:813.

2. Z Li, M Knetsch. Antibacterial strategies for wound dressing: preventing infection and stimulating healing. Curr Pharm Des 2018;24:936-51

3. W Xu, S Dong, Y Han, S Li, Y Liu. Hydrogels as antibacterial biomaterials. Curr Pharm Design 2018;24:843-54.

4. Sevanan M, Pongiya U, Peedikavil NJ. Antimicrobial susceptibility pattern of biofilm-producing Escherichia coli of urinary tract infections. Curr Res Bacteriol 2011;4:73-80.

5. Suman E, Jose J, Varghese S, Kotian MS. Study of biofilm production in Escherichia coli causing urinary tract infection. Indian J Med Microbiol 2007;25:305-6.

6. Clinical and Laboratory Standards Institute (CLSI). Performance Standard-Ninth Edition (M2-A9). Wayne, PA: Clinical and Laboratoey Standards Institute; 2006.

7. Micheal L Vasil. DNA microarrays in an analysis of quorum sensing: strengths and limitation; 2003. p. 0021-9193.

8. Fiorela Nievas, Pablo Bogino, Fernando Sorroche, Walter Giordano. Detection characterization and biological effect of quorum sensing signaling molecules in peanut-nodulating bradyrhizobia. Sensors (Basel) 2012;12:2851-73.

9. Mette E Skindersoe, Mortan Alhede, Richard Phipps, Liang Yang, Peter 0 Jensen, Thomas B Rasmussen, et al. Effects of antibiotics on quorum sensing in Pseudomonas aeruginosa. Antimicrob Agents Chemother 2008;52:3648-63.

10. Nathaniel C Cady, Kurt A Mckean, Jason Behnke, Roman. Inhibition of biofilm formation, quorum sensing and infection in pseudomonas aeruginosa by naturalproducts-inspired organosulfur compounds. Plos One 2012;7:e38492.

11. Ponnusamy $P$, Natarajan $V$, Sevanan $M$. In vitro biofilm formation by pathogenic escherichia coli and their antimicrobial susceptibility pattern. Asian Pac J Trop Med 2012;5:210-3.

12. Tajbakhsh E, Tajbakhsh S, Khamesipour F. Isolation and molecular detection of gram-negative bacteria causing urinary tract infection in patients referred to shahrekord hoospitals. Iran Iran Red Crescent Med J 2015;17:e24779.

13. Mittal S, Sharma M, Chaudhary U. Biofilm and multidrug resistance in uropathogenic Escherichia coli. Pathog Glob Health 2015;109:26-9.

14. Tajbakhsh S, Khamesipour F. Isolation and molecular detection of gram negative bacteria causing urinary tract infection in patients referred to shahrekord hoospitals. Iran Iran Red Crescent Med J 2015;17:e24779. 